\title{
Quantitative Effects of GABA and Bicuculline Methiodide on Receptive Field Properties of Neurons in Real and Simulated Whisker Barrels
}

\author{
HAROLD T. KYRIAZI, GEORGE E. CARVELL, JOSHUA C. BRUMBERG, AND DANIEL J. SIMONS \\ Departments of Neurobiology and Physical Therapy, University of Pittsburgh, Pittsburgh, Pennsylvania 15261
}

\section{SUMMARY AND CONCLUSIONS}

1. Carbon fiber multibarrel glass microelectrodes were used to record extracellular single-unit activity during microiontophoretic application of $\gamma$-aminobutyric acid (GABA) or bicuculline methiodide (BMI) onto layer IV barrel neurons in the somatosensory cortex of fentanyl-sedated rats. Excitatory and inhibitory aspects of the neurons' receptive fields were quantified with the use of controlled whisker stimuli. The principally activating whisker and one of its immediately adjacent neighbors were deflected alone or in paired combinations involving a condition-test paradigm.

2 . Units were distinguished electrophysiologically on the basis of the time course of their action potential waveforms. Data were obtained from 26 regular-spike units (RSUs; presumed spiny stellate cells) and 7 fast-spike units (FSUs; presumed GABAergic neurons ). An average of $15.0 \mathrm{nA}$ of GABA produced a one-third to one-half reduction in RSU responses evoked by the maximally effective stimulus. An average of $8.7 \mathrm{nA}$ of BMI was needed to counteract this reduction. This amount of BMI, in the absence of exogenous GABA, was found to increase average RSU and FSU responses by 98 and 53\%, respectively, relative to predrug levels.

3. For RSUs, the BMI-induced twofold increase in responses evoked by moving the principal whisker at the neuron's best deflection angle was accompanied by an almost threefold increase in responses evoked by similarly moving an adjacent whisker. Disproportionately large percentage increases were also seen for responses to nonpreferred directions of principal and adjacent whisker movement. BMI thus effectively increased receptive field size and decreased angular tuning. Similarly, responses to stimulus offsets, which are normally smaller than on responses, were increased proportionally more.

4. Predrug responses of FSUs were more vigorous than those of RSUs. However, FSUs showed a similar inverse relationship between percentage increase with BMI and initial response magnitude, although the proportional increases were less pronounced.

5. GABA, like BMI, had the greatest proportional effects on those responses that were initially smallest. It produced results opposite those of BMI, effectively decreasing receptive field size and sharpening angular tuning.

6. A previously described computational model of a barrel was tested for its ability to reproduce quantitatively the effects of BMI and GABA. The application of BMI was simulated by decreasing the strength of the inhibitory inputs onto the particular cell under study in the model network. GABA microiontophoresis was simulated by adding a constant hyperpolarizing voltage. The model RSUs and FSUs displayed proportional changes in response magnitude that were quantitatively similar to those of their biological counterparts.

7. Surround inhibition was greatly attenuated by BMI application, both for the real and simulated barrel neurons. Disinhibition was less pronounced for the former, perhaps because, unlike the simulated neurons, they also possess $\mathrm{GABA}_{\mathrm{B}}$ receptors, which are unaffected by BMI.

8. We conclude that the inhibitory receptive field properties of barrel neurons can be explained by intrabarrel inhibition and that the expansion of receptive field size and loss of angular tuning with BMI is due to an enhanced effectiveness of convergent, multiwhisker thalamocortical input. Examination of the model neurons' behavior suggests that the altered activity in response to GABA or BMI application, respectively, can be explained by the nonlinear effects of shifting somal membrane potential away from or toward the neuron's firing threshold.

\section{INTRODUCTION}

Cerebral cortical circuitry is characterized by a high density of excitatory synapses, many of which originate from local neurons within the cortex (White 1989). Synaptically mediated inhibition is thought to perform the important and probably related functions of controlling overall cortical excitability, helping to synchronize population activities, and enhancing temporal and spatial resolution within neuronal receptive fields. Thalamocortical axons synapse on both spiny (excitatory) and smooth (inhibitory) stellate neurons (White 1979), providing a means for continually matching locally generated inhibition with afferent excitatory drive (McCasland et al. 1992). In primary sensory areas, this aspect of cortical organization is manifested as a spatial overlap of stimuli that both strongly excite and strongly inhibit an individual neuron (Douglas et al. 1991; Ferster 1986; Gardner and Costanzo 1980; Simons 1985). Excitation and inhibition may not be entirely coextensive, however, because often a neuron's firing can be suppressed by stimuli presented outside the bounds of its excitatory receptive field (see Laskin and Spencer 1979).

Pharmacological blockade of inhibitory synaptic transmission by the $\gamma$-aminobutyric acid-A $\left(\mathrm{GABA}_{\mathrm{A}}\right)$ receptor antagonist bicuculline increases receptive field size in somatosensory cortex (Alloway et al. 1989; Dykes et al. 1984), expands output fields in motor cortex (Jacobs and Donoghue 1991), and broadens orientation tuning in visual cortex ( Sillito 1975). One interpretation of bicuculline's effects is that it blocks synapses that selectively counteract excitation produced by nonoptimal stimuli. Another is that, by decreasing $\mathrm{Cl}^{-}$conductance, bicuculline application leads to a general increase in cell excitability that in turn permits normally subthreshold excitatory inputs to be functionally expressed in the neuron's discharge. 
Electrophysiological studies of the rodent vibrissa system have demonstrated that cortical neuronal receptive fields, like those in other species, have both excitatory and inhibitory components. Layer IV of the somatosensory cortex contains morphologically identifiable neuronal groups, called 'barrels,' that correspond to individual mystacial vibrissae (Welker 1976; Woolsey and Van der Loos 1970). Within a barrel, the majority of electrophysiologically studied neurons have well-focused receptive fields. Neurons respond much more vigorously to deflections of the anatomically appropriate vibrissa, called the principal whisker $(\mathrm{PW})$, than to deflections of the immediately adjacent ones (ArmstrongJames and Fox 1987; Simons and Woolsey 1979), and excitatory responses evoked by deflecting one vibrissa are suppressed by prior deflection of a neighboring one (Simons 1985 ). Neurons in the ventrobasal thalamic "barreloids" typically have larger excitatory receptive fields, whereas inhibitory interactions among nearby whiskers are observed less often there. These and other differences between the response properties of thalamic and cortical neurons lead us to conclude that a balance of excitatory and inhibitory influcnces of the barrel circuitry actively regulates functional characteristics of layer IV neurons, including receptive field size (Simons and Carvell 1989).

A barrel contains a network of interconnected spiny and smooth cells, and both cell types receive excitatory thalamocortical synapses (Agmon and Connors 1992; Keller 1995). Parametric studies of thalamocortical response transformations in a computer-simulated barrel (Kyriazi and Simons 1993 ) suggest that synaptic connections among and between excitatory and inhibitory cells, representing positive and negative feedback, act in conjunction with neuronal nonlinearities to enhance response differentials between strong and weak input signals. An example of the former is responses of thalamic relay neurons evoked by the onset of PW movement at a preferred angle; weaker thalamocortical signals are produced by deflection of an adjacent whisker at a nonoptimal angle. A direct prediction of the model is that blockade of a barrel neuron's $\mathrm{GABA}_{\mathrm{A}}$ receptors should, by making the cell more responsive, enlarge its receptive field, reduce its directional tuning, and increase the size of its OFF response relative to its ON response. Converse effects should accompany increased synaptic inhibition.

In the present study we test these predictions using microiontophoresis of GABA and bicuculline methiodide (BMI). Effects were quantified for two types of previously characterized units, regular-spike units (RSUs), which are presumed spiny barrel neurons, and fast-spike units (FSUs), which are thought to be GABAergic smooth cells ( see McCormick et al. 1985). Electrophysiological data were compared with data obtaincd from the model's corresponding spiny and smooth barrel neurons, which either were subjected to a constant hyperpolarizing voltage (simulating GABA) or had their incoming inhibitory synaptic weights decreased (to simulate the action of BMI). Results obtained for real and simulated neurons were highly similar. Together, the findings demonstrate that GABA disproportionately suppresses, and BMI disproportionately enhances, responses to inputs that are normally only weakly effective. In this manner, inhibition increases signal-to-noise ratios. Thus control of neuronal excitability by GABA-mediated inhibition is an effective mechanism for improving stimulus discrimination.

\section{METHODS}

\section{Animals}

Twelve adult female rats weighing 200-350 g were used for the experiments (Sprague-Dawley strain; Zivic-Miller Laboratories, Allison Park, PA). Surgical procedures for preparing the animals for cortical unit recordings were described previously (Simons and Carvell 1989). Briefly, under halothane anesthesia, a small opening was made in the skull and dura overlying the face area of the primary somatosensory cortex, and an acrylic dam was constructed around the craniectomy. A steel post was embedded in the dental acrylic and was used to hold the animal's head thereafter. After all surgical procedures were completed, halothane was discontinued and the animal received a continuous intravenous infusion of fentanyl, a synthetic opiate receptor agonist (Sublimaze, Janssen Pharmaceuticals; $5-10 \mu \mathrm{g} \cdot \mathrm{kg}^{-1} \cdot \mathrm{h}^{-1}$ ). This maintained the rats in a lightly narcotized and sedated state during the recording experiment. Animals were paralyzed by pancuronium bromide $\left(\sim 1.6 \mathrm{mg} \cdot \mathrm{kg}^{-1} \cdot \mathrm{h}^{-1} \mathrm{iv}\right)$, artificially respired through a tracheal cannula using a positive pressure respirator, and kept warm via a servo-controlled heating blanket. The animal's condition was assessed by monitoring the electroencephalogram, pupillary reflexes, femoral arterial blood pressure, heart rate, and airway pressure waveform. An experiment was terminated if normative physiological conditions could not be maintained.

At the conclusion of the experiment, the animal was administcred a lethal dose of pentobarbital sodium and perfused for horseradish peroxidase (HRP) and cytochrome oxidase (CO) histochemistry (Simons and Land 1987). Frozen sections of the right hemisphere were cut in a tangential plane so that the barrel field could be readily visualized, and alternate $60-\mu \mathrm{m}$ sections were taken for HRP and CO processing. Electrode tracks and recording locations were reconstructed using microdrive depth readings, maps of surface blood vessels and electrode penetration sites, and small marks made during the experiment by iontophoretic ejection of HRP at selected recording locales.

\section{Microelectrodes}

Seven-barrel glass micropipettes with a carbon fiber recording channel were used for simultaneous extracellular unit recordings and microiontophoresis. The microelectrodes were made using methods described by K. D. Alloway (personal communication, and see Alloway and Burton 1986). The end of a length $(>10$ $\mathrm{cm}$ ) of 0.011-in.-diam stainless steel wire was coated with conductive paint (Silver Print; GC Electronics, Rockford, IL) and quickly touched end-to-end ( $\sim 5 \mathrm{~mm}$ overlap) to a single carbon fiber extending from a 10- to 15-cm-long bundle of carbon fibers ( $7 \mu \mathrm{m}$ diam; Fiber Matcrials, Biddeford, ME). After drying (1-2 min), the fiber was pulled straight away from the fiber bundle via the wire and was loaded, wire end first, into the center barrel of a seven-barrel assembly of glass microfilament-containing capillary tubes $(10 \mathrm{~cm}$ long $\times 1.0 \mathrm{~mm}$ OD for each capillary tube; World Precision Instruments, Sarasota, FL). Thin strands, 2-3 mm long, of deKhotinsky cement (Sealstix; Thomas Scientific) were placed adjacent to the wire just inside the capillary tube and were melted, fixing the wire and attached carbon fiber in place. Excess wire was trimmed, leaving $\sim 7 \mathrm{~mm}$ protruding from the end of the glass; this was used for making electrical contact with the carbon fiber.

The seven-barrel capillary tube assembly was loaded into a vertical pipette puller (Narishige), wire end down, and pulled, in a single pull, to produce a gradual taper; at $1.8 \mathrm{~mm}$ from the tip, the electrode was no more than $0.25 \mathrm{~mm}$ diam. Excess carbon fiber 
was cut away, leaving $\sim 5 \mathrm{~mm}$ protruding from the glass tip. Under a compound microscope, the fiber tip was electrochemically etched (50-70 $\mu \mathrm{A}$ alternating current) to a pencil-point shape with the use of a drop of potassium dichromate/sulfuric acid solution (1 $\mathrm{ml}$ of $10 \mathrm{mg} / \mathrm{ml} \mathrm{K}_{2} \mathrm{Cr}_{2} \mathrm{O}_{7}$ plus $200 \mu \mathrm{l}$ concentrated sulfuric acid) suspended on a wire loop (after Armstrong-James et al. 1980); residual etching solution was removed by passing the fiber through a droplet of water. A heating filament coil was placed around the back end of the assembly and was heated to melt the deKhotinsky cement. With the use of a micromanipulator and $\times 200$ magnification, the wire and attached carbon fiber were pulled back until the etched tip protruded $5-10 \mu \mathrm{m}$ from the tip of the glass micropipette, which had a diameter of $\sim 12 \mu \mathrm{m}$. The heat was then turned off, recementing the wire and carbon fiber in place. The resistance of the carbon fiber electrode was $\sim 1 \mathrm{M} \Omega$. Electrodes were usually used within 1 wk after having been pulled. No systematic study was made of the rate of tip deterioration, but on a few occasions pipettes in storage for up to several months were used, and these gave satisfactory results.

Iontophoretic solutions were loaded into the other microelectrode barrels on the day of use. One barrel contained a $10 \%$ solution of HRP in $50 \mathrm{mM}$ tris (hydroxymethyl) aminomethane $\mathrm{HCl}$ buffer, $\mathrm{pH}$ 6.8, for use in marking selected recording sites (Simons and Land 1987). Other barrels contained 0.2 M GABA ( $\mathrm{pH}$ adjusted to 3.0 with $\mathrm{HCl}$; Sigma Chemical), BMI $(2.5 \mathrm{mM}$ in $0.9 \% \mathrm{NaCl}$, pH 2.5-3.0; Sigma Chemical), or $0.9 \% \mathrm{NaCl}$ for current balancing. Typically, two barrels were filled with GABA and two with $\mathrm{BMI}$; the additional barrel could serve as a reserve in the event that the other became clogged and unable to pass current. Resistance of iontophoretic channels was $\sim 22 \mathrm{M} \Omega$.

\section{Whisker stimulation}

At the beginning of the experiment, a tungsten microelectrode $(\sim 10 \mathrm{M} \Omega$, Frederick Haer, Brunswick, ME) was inserted into the cortex at the craniectomy site, and multiunit recordings were used to identify the probable location of a barrel center. Electromechanical stimulators were subsequently attached to the PW and to one to four of its immediately adjacent neighbors (Simons 1983). Each stimulator was inserted over the distal end of a whisker, which was first shortened to a length of $10-12 \mathrm{~mm}$. The stimulators produced ramp-and-hold movements having an amplitude of 1.0 $\mathrm{mm}$, onset and offset velocities of $\sim 125 \mathrm{~mm} / \mathrm{s}$, and duration of $200 \mathrm{~ms}$. Because responses of cortical neurons, like those of the primary afferent fibers that innervate the vibrissae, depend on the angular direction in which a whisker is moved, deflections were made in eight directions (in $45^{\circ}$ increments relative to the horizontal alignment of whisker rows ).

PROTOCOL 1. To assess aspects of a unit's excitatory receptive field, the PW and one to four of the immediately adjacent whiskers were moved one at a time in randomized sequence. The battery of stimuli consisted of 10 sets of movements in the eight directions, giving 80 repetitions for each whisker; interstimulus intervals were $1.5 \mathrm{~s}$. The average discharge during a 20 -ms period following movement onset (i.e., ON response) was computed on-line for each direction, and polar plots obtained for the different whiskers were displayed on a graphics terminal.

PROTOCOL 2. We assessed aspects of a unit's inhibitory receptive field by deflecting pairs of whiskers in a condition-test paradigm (Simons and Carvell 1989). Here, the PW and one of the adjacent whiskers were moved one before the other, with a 30 -ms delay between the two stimulus onset (and offset) times. The test stimulus was moved at the maximally responsive deflection angle for that whisker's on response, determined initially using protocol 1 , whereas the conditioning stimulus was moved in 10 sets of randomized sequences of the eight deflection angles. The test stimulus was also delivered 10 times by itself, yielding 90 stimulus presentations per pair; interstimulus intervals were $1.5 \mathrm{~s}$. The $\mathrm{PW}$ and up to four adjacent whiskers served alternately as the test and conditioned stimuli, so that as many as eight pairings were tested.

Together, protocols 1 and 2 required $>25 \mathrm{~min}$ when all four adjacent whiskers were stimulated. Consequently, after the initial experiments established that drug-induced changes in a given unit's responses were at least qualitatively similar for the four adjacent whiskers, we restricted our study to the PW and the caudally adjacent whisker, which has been found previously to exert the strongcst inhibitory effects of the PW's immediate neighbors (see McCasland et al. 1991).

\section{Electrophysiological recordings and microiontophoresis}

The multibarrel microelectrode was advanced through the tissue with the use of a hydraulic microdrive attached to a stepping motor while a stimulator was used to move the $\mathrm{PW}$ in different directions. Stimulus-driven units were isolated using an amplitude discriminator (WP Instuments), and their receptive ficld characteristics were assessed using the protocols described above. High- and low-pass frequency filters were set at $300 \mathrm{~Hz}$ and $10 \mathrm{kHz}$, respectively. GABA and BMI were applied iontophoretically (Neuroprobe, Medical Systems). Drug delivery was standardized as follows. The PW was repeatedly deflected in the unit's preferred direction while ejection currents for GABA were slowly increased to a level where the unit's response to deflection onset was reduced by one third to one half. This was monitored using a computer program that displayed a running average of the preceding 10 responses. BMI ejection currents were then titrated to restore the response, with GABA still present, to its predrug, control level. The GABA current was then halted, maintaining that of BMI. With this BMI dose, the response of the unit generally increased to approximatcly twice its control value ( see below). This was not a saturating dose because, when tested, it was found that considerably greater increases in unit discharge could be produced with the use of larger BMI ejection currents. Unit activity typically returned to predrug levels within $10 \mathrm{~min}$ of the cessation of drug delivery. Retaining currents of $-15 \mathrm{nA}$ were applied to drug barrels not in use.

\section{Data analysis}

An LSI $11 / 73$ computer was used to control the whisker stimulation and to collect data, which were later analyzed using either the $11 / 73$ or a personal computer running a data base and statistical package (SPSSPC + , SPSS). Interspike intervals occurring during a 500-ms period of data collection bracketing the whisker stimulus were measured with a resolution of $100 \mu$ s and stored sequentially on disk so that spike trains for individual stimulus presentations could be reconstructed. These were converted into peristimulus time histograms (PSTHs) having 1-ms bins. The mean and variance of spike discharges for selected time periods were derived from the individual spike trains. Responses to stimulus onsets (ON) and offsets (OFF) were computed for a 20 -ms period after the beginning of whisker movement away from or back to its resting position. Background activity, hereafter referred to as "spontaneous activity,' was measured during a 100-ms period just before stimulus onset. Stimulus-evoked response values were not corrected for such spontaneous activity.

For protocol 2, we first computed the conditioned-test response averaged over all eight angles of the conditioning stimuli. Condition-test ratios were then computed by dividing the conditionedtest response (in spikes per stimulus) by the response to the test stimulus alone; a value of 1.0 represents no effect of the preceding whisker deflection and 0.0 represents complete suppression of the test response. Because the choice of the test stimulus was based on the test whisker's maximally effective on response angle, data 
were so analyzed only for stimulus onsets. Ratios were computed separately for predrug and BMI conditions, thus taking into account the increased PW responsiveness under BMI.

In this paper we report data analyses for units whose recording sites were found, on histological reconstruction, to be located within the CO-rich centers of the layer IV barrels. We distinguished two types of previously described units on the basis of digitized samples of their electrical waveforms. The criteria used were the $10-90 \%$ rise times of the first positive-going peak (for spikes with initially negative waveforms ) and the width of this peak at its halfheight. The product of these two measures yielded nonoverlapping populations of shorter- and longer-duration waveforms (cutoff value of $0.025 \mathrm{~ms}^{2}$ ). The former were similar to the action potentials of FSUs recorded with the use of glass micropipettes (e.g., Kyriazi et al. 1994), and the latter were similar to those of previously described RSUs. A total of 26 RSUs and 7 FSUs were studied quantitatively in 12 experiments. In many of the animals, units were also recorded at other cortical depths; those results will be presented in a later report.

A measure of directional selectivity called the "tuning ratio", was defined as the oN response at the maximally responsive deflection angle divided by the average response at all eight angles. A value of 1.0 would mean the cell's PW responded equally well at all deflection angles; a value of 8 (the highest possible value) would mean the neuron responded only at one angle.

\section{Computer simulations}

The computational model has been described in detail (Kyriazi and Simons 1993). Briefly, it consists of a network of 100 simulated barrel neurons - 30 inhibitory, smooth cells (simulated FSUs) and 70 excitatory, spiny cells ( simulated RSUs) ${ }^{1}$ - whose individual connections and connection strengths were set stochastically, so that only the means and SDs are specified. Each neuron is activated by spike trains from previously recorded thalamocortical neurons that were studied using the same whisker stimulation protocols employed here. The model neurons temporally integrate and linearly sum their excitatory (positive) and inhibitory (negative) inputs, and the net membrane potential is converted into a probability that an action potential will occur. The neurons have two nonlinear properties (absolute refractory periods and sigmoid spike activation functions), and these differ for spiny and smooth cells, rendering the former more nonlinear with regard to their input/ output behavior. The simulated barrel neurons produce spike train output that can be compared quantitatively with results obtained in electrophysiological studies.

In the original modeling study, the computer's random number generator, which was used to set network weights and connectivities stochastically, was itself seeded randomly, producing subtly different networks for each simulation. In the present study we wanted to examine the responses of individual neurons before and during simulated GABA or BMI application. Therefore the random number generator seed was fixed so that a single instantiation of the network could be reproduced exactly, and the same cells could be examined under various simulation conditions.

Microiontophoresis of GABA or BMI was simulated, respectively, by adding a constant hyperpolarizing voltage to a neuron or by decreasing the strength ("weight") of the inhibitory synapses onto it; no other changes were made to any other neuron in the network. For computational ease, the simulated drug dosagcs were not titrated individually for each neuron, but were instead applied equally to all (one at a time). For BMI, a $70 \%$ decrease

\footnotetext{
${ }^{1}$ For the remainder of the article, the electrophysiologically derived terms RSU and FSU refer to the real neurons, whereas the anatomically derived names spiny and smooth cells refer, respectively, to the model RSUs and FSUs.
}

in inhibitory synaptic weight was found to produce, on average, an approximately twofold increase in the maximal angle on response of spiny neurons, the same increase that was sought and achieved in the electrophysiologically studied RSUs. Although all of the simulated neurons displayed increased activities with this manipulation, 7 of the 70 spiny cells received so few inhibitory inputs, because of the stochastic nature of the network connectivity, that the increases were much less than twofold. Because we wishcd to make quantitative comparisons between real and simulated neurons, we excluded data from seven spiny cells that displayed increases of $<1.5$-fold. Similarly, the responses of 2 of 30 simulated smooth cells were excluded (cutoff $=1.2$-fold). All of the neurons in the simulated barrel were included in the GABA analyses, because the effect of membrane hyperpolarization (the simulated GABA) was independent of the number of inhibitory inputs the cells received.

Computer simulations were performed on a CRAY supercomputer at the Pittsburgh Supercomputing Center, using the same FORTRAN program and the identical parameter settings previously described (Kyriazi and Simons 1993). Spike trains were analyzed identically to those of the electrophysiologically studied units.

\section{RESULTS}

\section{General observations}

Microiontophoretic application of GABA led to a clear decrease in unit responsiveness in 17 of 20 cases tested; the remaining 3 units showed no obvious change with 30-nA currents, the maximum that we used. Paradoxically, some neurons responded to GABA application with an initial increase in spontaneous and stimulus-evoked activity, but this effect was short-lived, usually $<30 \mathrm{~s}$, and was not quantified. $^{2}$

Figure 1, $A$ and $B$, shows the effect of GABA on an RSU recorded in the $\mathrm{C} 2$ barrel. Averaged over all eight deflection angles, this unit, in the predrug condition, responded with 1.31 spikes per stimulus onset to deflections of $\mathrm{C} 2$, the $\mathrm{PW}$. Smaller ON responses were evoked by two adjacent whiskers [C1 (0.51 spikes per stimulus) and D2 (0.26 spikes per stimulus)], and the other two whiskers elicited virtually no excitatory response. Application of $9 \mathrm{nA}$ of GABA reduced the PW response to 0.81 spikes per stimulus (a $38 \%$ reduction) and the $\mathrm{C} 1$ response to 0.14 spikes per stimulus (a $73 \%$ reduction ); the $\mathrm{D} 2$ response was almost entirely eliminated, as was the neuron's spontaneous activity. The C2 OFF response was reduced by $55 \%$ and that of $\mathrm{C} 1$ was reduced by $>90 \%$. Thus GABA disproportionately reduced stimulusevoked responses that were initially smaller, and it virtually eliminated spontaneous activity.

As shown in Fig. 1C, BMI produced opposite effects on this neuron. With a titrated dose of $13 \mathrm{nA}$, overall excitability increased as evidenced by the greater spontaneous and stimulus-evoked responses. The PW on response increased $124 \%$, to 2.93 spikes per stimulus, and the smaller PW ofF response showed an even greater relative increase (0.75-1.99 spikes per stimulus: a $165 \%$ increase). Similarly, responses to $\mathrm{C} 1$ and D2 deflection onsets increased 276 and 338\%, respec-

\footnotetext{
${ }^{2}$ Similar short-lived depolarizations have been reported for dendritic application of GABA (Connors et al. 1988), and cell firing induced by such application is rapidly abolished by higher concentrations of GABA (Andersen et al. 1980).
} 
A Pre-Drug

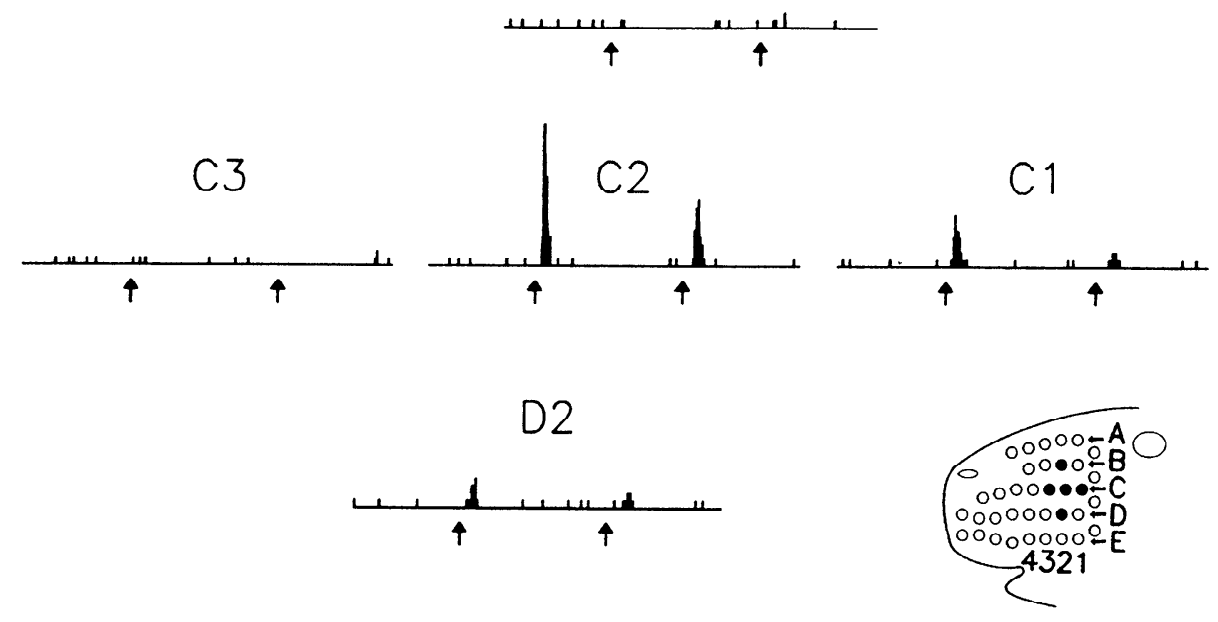

B2

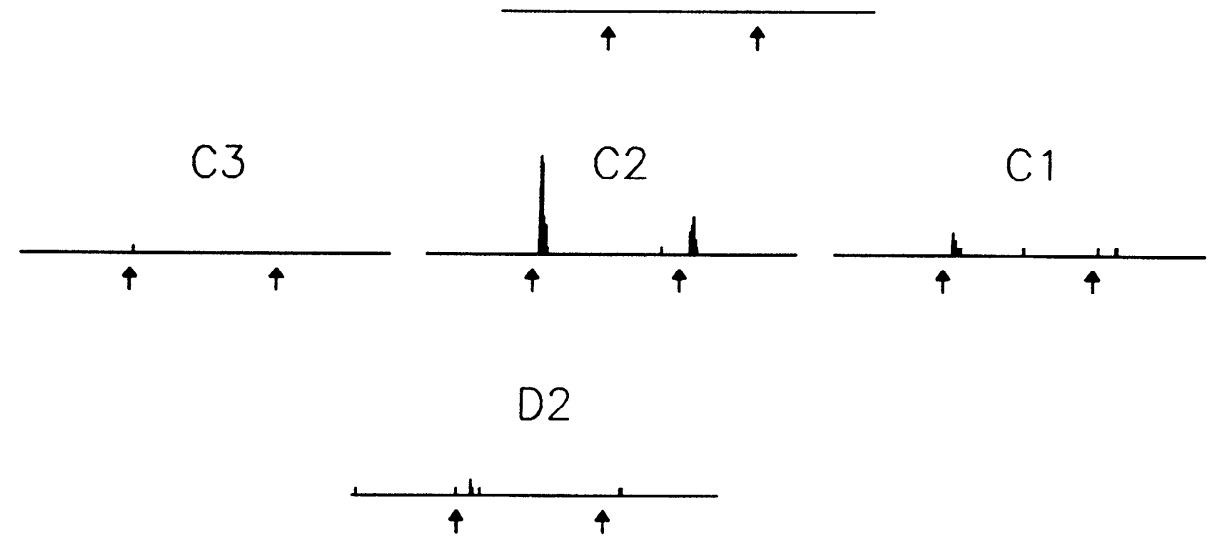

FIG. 1. Effect of $\gamma$-aminobutyric acid (GABA) on the excitatory receptive field characteristics of a barrel regular-spike unit (RSU). The unit was located $888 \mu \mathrm{m}$ below the pial surface, within the $\mathrm{C} 2$ barrel. Peristim ulus time histograms (PSTHs) include the spikes occurring during 10 sets of deflections of the indicated whisker at each of 8 angles ( 80 stimuli total, l-ms bins). Arrows: beginnings of the onsets and offsets of whisker movement during the 500-ms data collection period. $A$ : control, predrug response to deflection of $\mathrm{C} 2$, the principal whisker ( $\mathrm{PW}$ ), and its 4 nearest neighbors. $B$ : as in $A$, but under the influence of $9 \mathrm{nA}$ of GABA. $C$ : same neuron, with $13 \mathrm{nA}$ bicuculline methiodide (BMI). Inset: deflected whiskers. Scale at bottom right applies to all PSTHs.

\section{C $\mathrm{BMI}$}
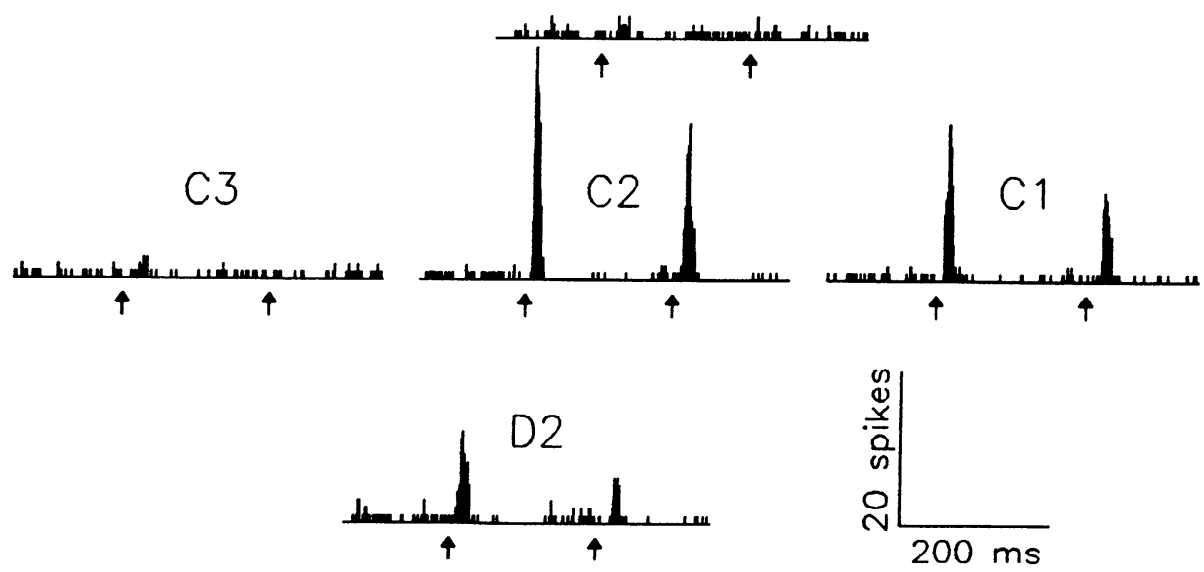

tively. Small, longer-latency responses to $\mathrm{C} 3$ and $\mathrm{B} 2$ became more apparent.

The specificity of neuronal responses to deflections of whiskers in different directions was also reduced by BMI. Figure 2 shows PSTHs and the corresponding polar plot obtained by deflecting an RSU's PW in eight directions. In 


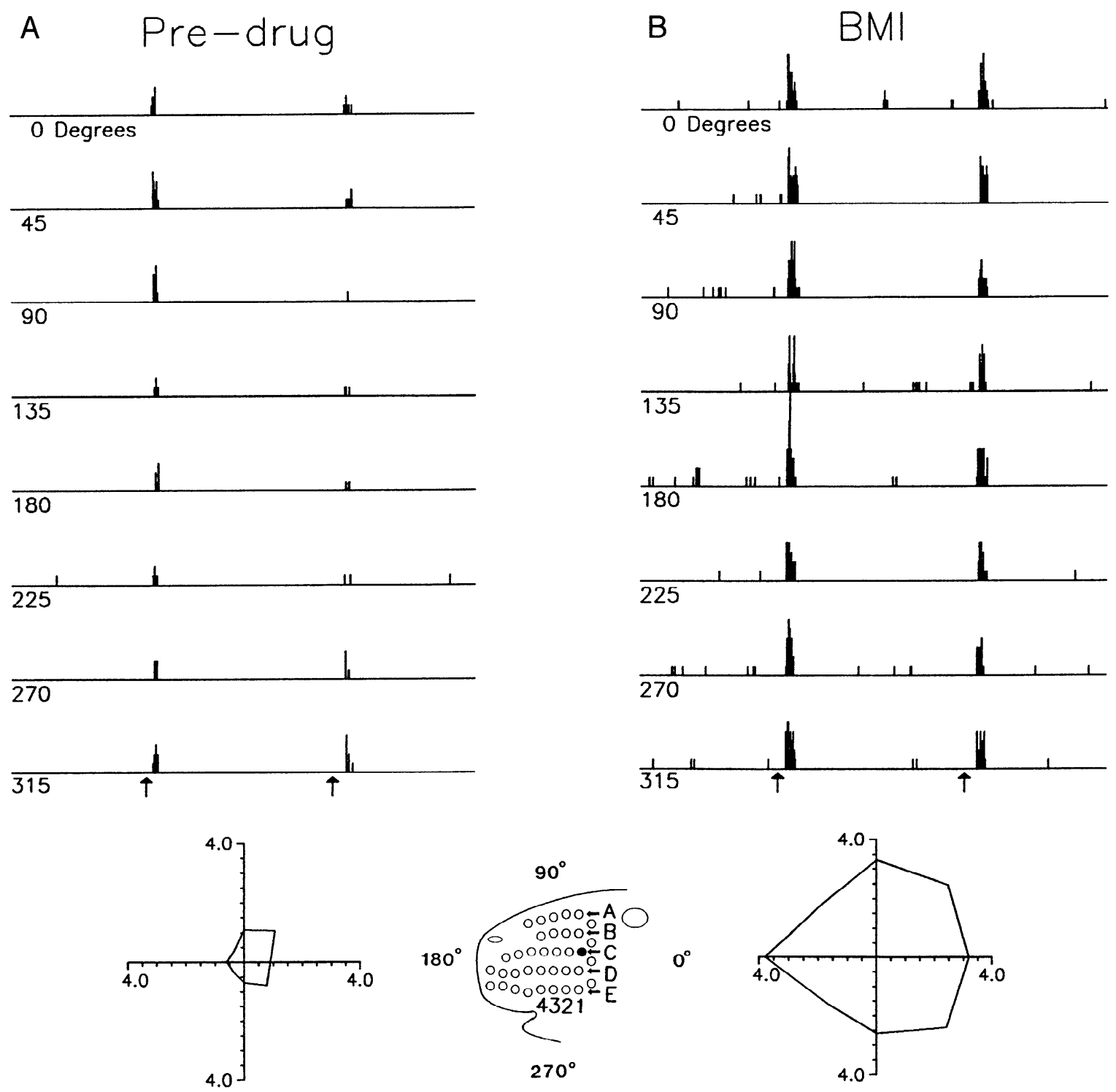

FIG. 2. Effect of BMI on angular tuning of an RSU. The unit was in the $\mathrm{C} 1$ barrel, at a depth of $736 \mu \mathrm{m}$. PSTHs show the summed responses to 10 deflections of the unit's PW at each of 8 angles; $0^{\circ}$ represents a caudal deflection onset, $90^{\circ}$ an upward one (see inset). Arrows: beginnings of stimulus onsets and offsets. Polar graphs at bottom plot the magnitudes of the average $\mathrm{ON}$ responses in spikes per stimulus at the various angles. Each $X$-axis contains 5001 -ms bins. All vertical axes have a scale maximum of 10 spikes per bin; the $180^{\circ}$ oN response in $B$ contains a 10-spike bin. $A$ : data obtained before application of BMI. $B$ : data obtained during application of $20 \mathrm{nA}$ of BMI.

the predrug condition, the unit displayed a preference for dorsal/caudal whisker movements (stimulus onsets); the strongest response was 1.50 spikes per stimulus onset, and the average of all eight directions was 0.86 . With BMI, the unit's overall responsiveness increased such that the maximal angle on response was 3.80 spikes per stimulus and the average of all eight was 3.09. Compared with the predrug condition, the overall shape of the polar plot is more circular, reflecting the fact that larger increases were obtained with stimulus directions that were initially less effective. For this unit the tuning ratio (see METHODS) is 1.74 in the predrug condition versus 1.23 with BMI.

Figure 3 shows data for a simulated spiny barrel neuron whose initial degree of angular tuning (tuning ratio of 1.76) was similar to that of the RSU of Fig. 2. Reducing the strength of the inhibitory synaptic connections onto this cell, to simulate BMI application, caused a $150 \%$ increase in the maximal angle on response and even greater increases at the initially less responsive directions; note, for example, the appearance of oN responses at 270 and $315^{\circ}$. As a result, the unit's tuning ratio decreased from 1.76 to 1.32 .

\section{Quantitative comparisons of drug effects on real and simulated barrel neurons}

To be able to compare data from different neurons, in early expcriments we titrated BMI application against ejection currents of GABA that produced a one-third to one-half reduction in PW ON responses at each neuron's most effective stimulus angle. For RSUs $(n=11)$ this required GABA currents of $15.0 \pm 8.0(\mathrm{SD}) \mathrm{nA}$; for FSUs $(n=5), 20.8 \pm$ $10.0 \mathrm{nA}$ were used. The titrated level of BMI ( $8.7 \mathrm{nA}$ for 

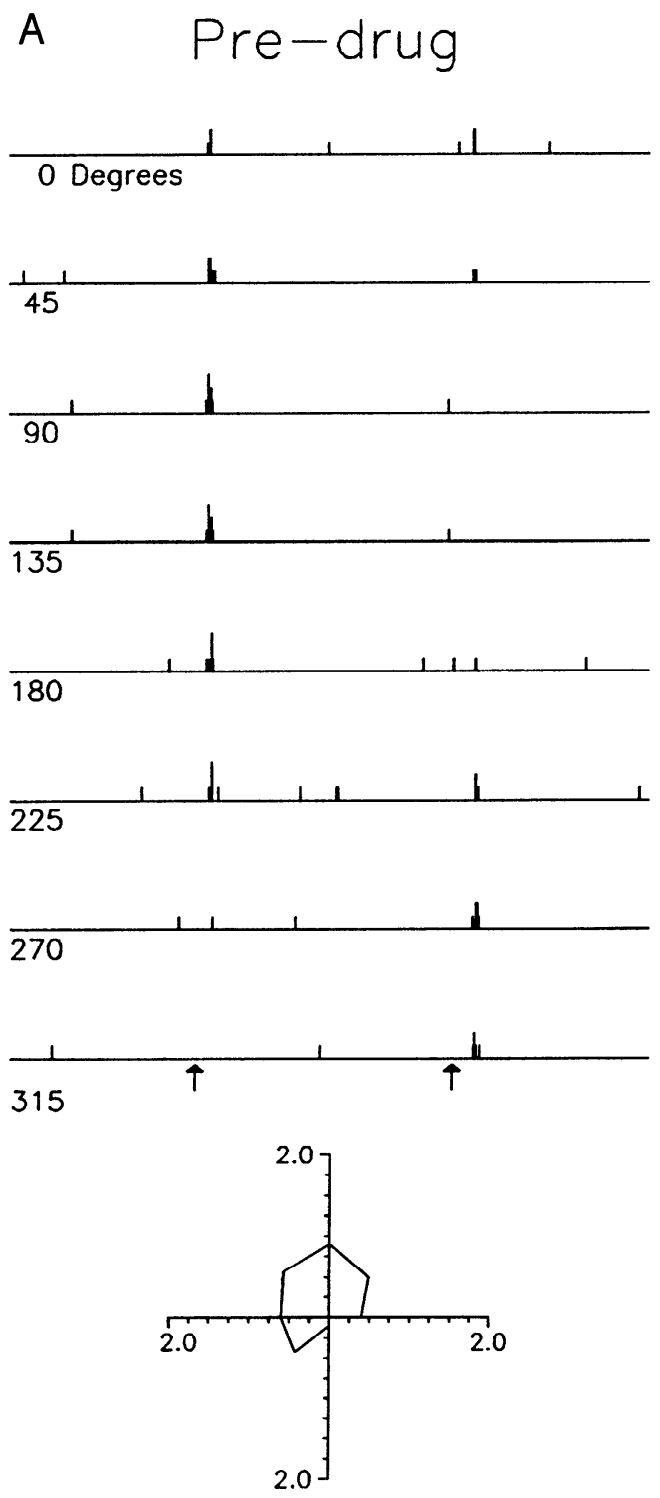
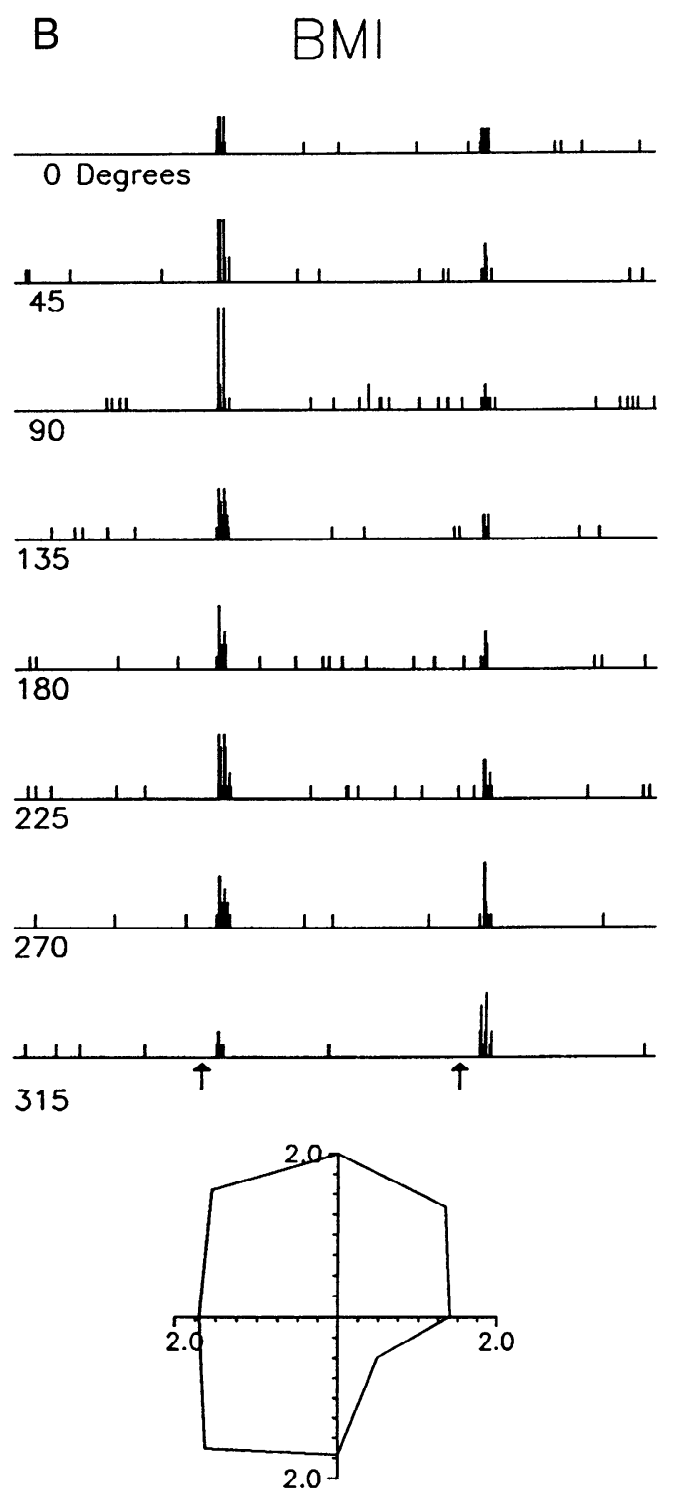

FIG. 3. Effect of simulated BMI application on angular tuning of a simulated RSU. Conventions and scaling as in Fig. 2.

RSUs ) typically produced slightly less than a doubling of the original response. Subsequently we dispensed with the GABA titration, simply applying enough BMI to produce an increase of $\sim 100 \%$. We obtained quantitative receptive field data for $23 \mathrm{RSUs}$, in which on average $8.8 \pm 5.2 \mathrm{nA}$ were used to attain the desired doubling of the PW maximum angle on responses. When we attempted to double the response of FSUs, the spontaneous activity, which in these units is high to begin with, became so prominent that we were concerned that it might interfere with the interpretation of stimulus-evoked data, even if corrected by subtraction. Consequently, we used BMI currents $(8.1 \pm 5.4, n=7)$ similar to those used for RSUs; this produced an average increase in FSU maximum angle oN responses that was roughly one-half that of RSUs $(53 \%)$. For simulations, inhibitory synaptic weights onto model spiny neurons were reduced to achieve an approximate doubling of their maximum angle $\mathrm{PW}$ on response; this required a $70 \%$ reduction in synaptic weight. Interestingly, this same percentage reduction, when used for model smooth cells, yielded an increase $(46 \%)$ close to that observed in FSUs.
Figure 4 shows average changes in angular tuning produced by BMI application. RSUs are more selective for deflection angle than FSUs. With BMI, both types of units displayed a decrease in directional selectivity, but the changes were more pronounced for the RSUs. Similarly, on a unit-by-unit basis, RSUs that were the most selective to begin with also showed the largest decrease in tuning with BMI application, and this relationship was highly significant ( $r=0.90, P<0.0001$ ). Data from the model neurons are similar in that tuning decreases with reductions in inhibitory synaptic strengths and effects are more pronounced for spiny cells. Both classes of simulated neurons showed greater effects than their biological counterparts. This may reflect the nature of the model's intrinsic connectivity, wherein any neuron can contact any other. A lack of specificity in excitatory feedback connections would provide heterogeneous inputs, many of which are normally subthreshold.

Effects of BMI-induced changes in PW and adjacent whisker responses are quantified for 23 RSUs and 7 FSUs in Fig. 5. Responses to stimulus onsets and offsets were com- 


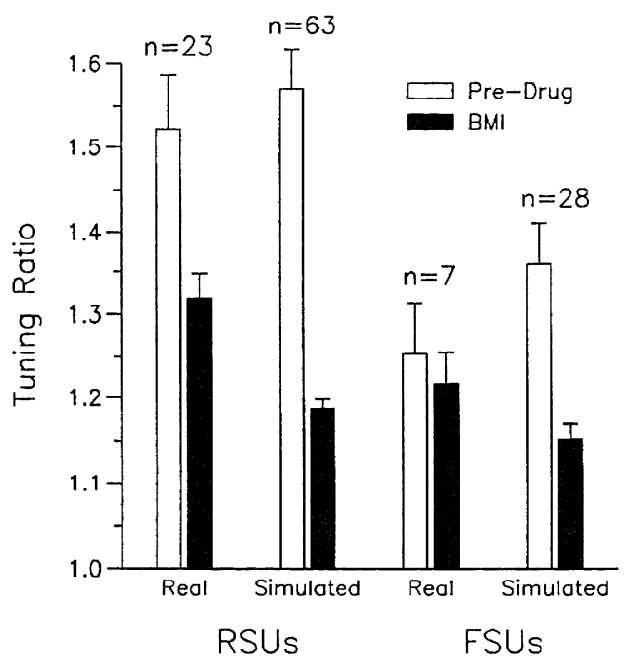

FIG. 4. Effect of BMI on tuning of real and simulated RSUs and fastspike units (FSUs). The tuning ratio is the maximal angle ON response divided by the on response averaged over all 8 deflection angles. Plotted are the means $\pm \mathrm{SE}$, with $n$ values as indicated. puted separately, and data are organized on the absicssa in order of descending average response magnitude. For RSUs, the onset of PW deflections in the maximally effective direction evoked the largest responses in predrug and BMI conditions, whereas stimulus offsets applied to adjacent whiskers evoked the smallest. Comparison of predrug and BMI values indicates that the drug produces greater relative increases in responses evoked by the initially less effective stimuli. This disproportionate effect is more pronounced in RSUs than in FSUs, whose overall predrug activity levels are higher. BMI application also increased spontaneous activity. Relative increases were greater for RSUs than FSUs, and this difference parallels the stimulus-evoked data in that RSU spontaneous activity is lower to begin with (note difference in scales).

Data from Fig. 5 were used to compute proportional increases in activity induced by BMI. Each point in Fig. 6 represents the ratio of average post-BMI to pre-BMI activity for each response, plotted versus the average pre-BMI magnitude of that particular response (e.g., maximal angle PW ON). Figure 6 also includes ON and OFF responses averaged over all eight deflection angles for both the PW and adjacent
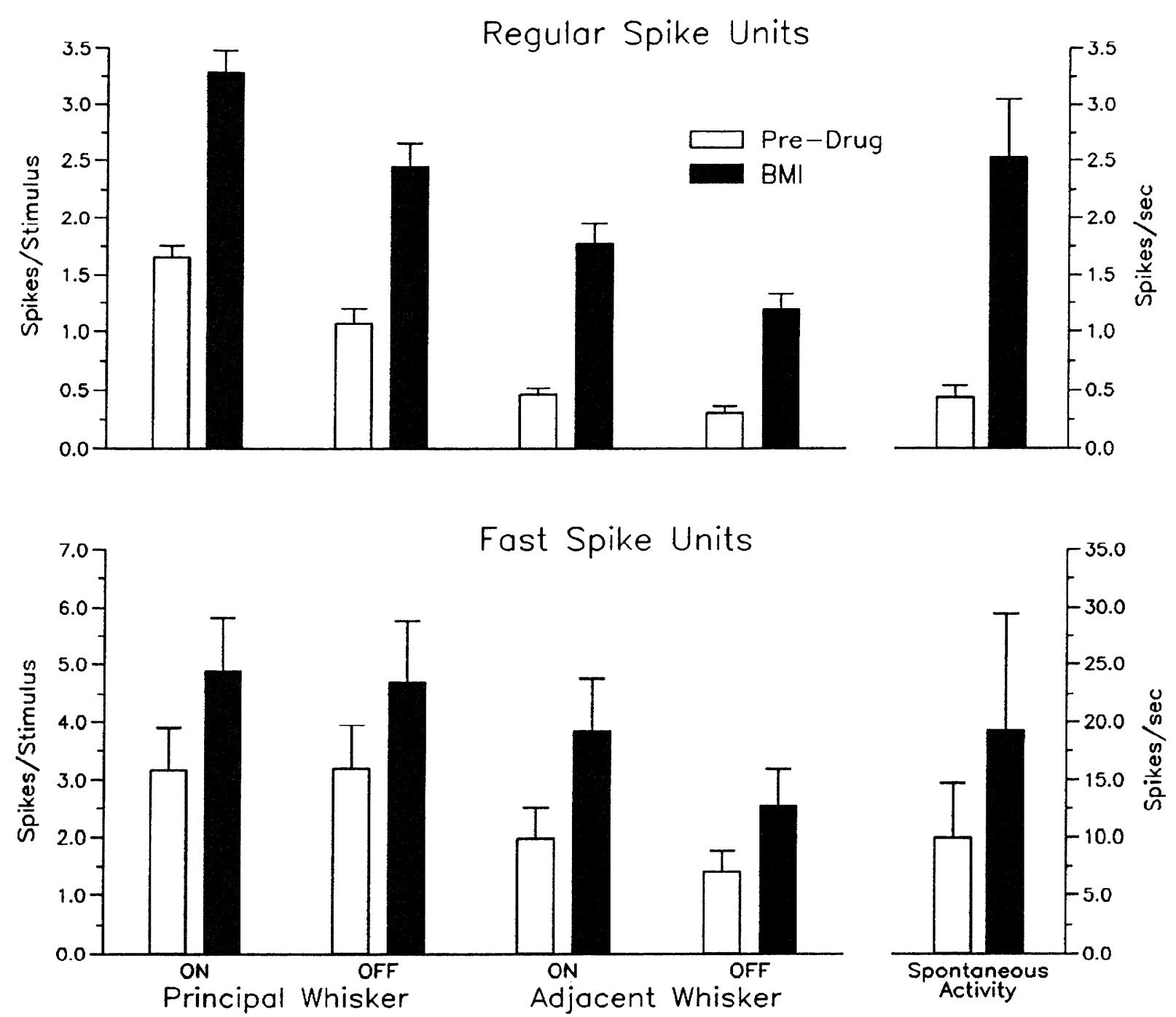

FIG. 5. Effect of BMI on RSU and FSU spontaneous activity and responses evoked by the PW and adjacent whisker deflection onsets and offsets at their best deflection angle. Values are the means \pm SE. For RSUs, $n=23$; for FSUs, $n=$ 7. Note differences in the 4 vertical scales. 


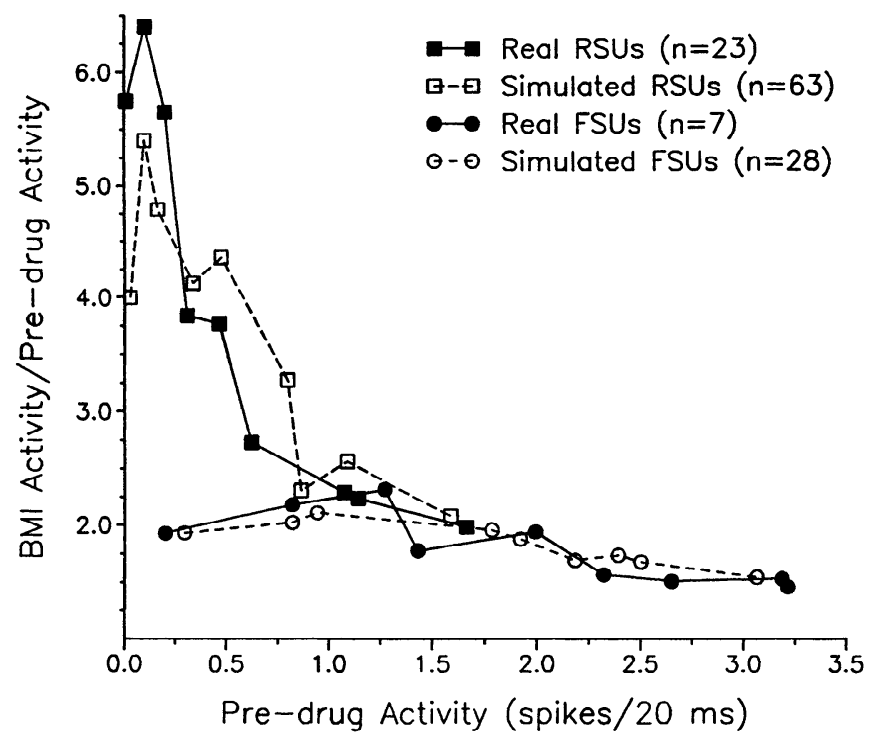

FIG. 6. BMI-induced changes in activity levels of real and simulated RSUs and FSUs. Points along the vertical axis: ratios of the mean values for each population. The 9 points plotted for each group varied somewhat in their order along the abscissa for the 4 groups. The 3 leftmost data points of each curve are spontaneous activity, adjacent whisker OFF response averaged over all 8 angles, and adjacent oN averaged over all 8 angles, in that order, and the 2 rightmost points are the PW maximal angle ON and OFF responses. The central 4 points consist of, in varied order, adjacent maximal angle ON and OFF responses and PW ON and OFF responses averaged over all 8 angles. In generating the simulated data, the extent of disinhibition $(70 \%)$ was chosen so that the rightmost data point for the spiny cells (maximal angle on response) gave the desired doubling of activity; all other data points constituted independent variables.

whiskers. Response magnitudes are ordered from smallest to largest on the abscissa (see legend). Also shown are curves derived from 63 simulated spiny cells and 28 simulated smooth cells. These analyses clearly demonstrate that, for both real and simulated neurons, BMI disproportionately enhances initially weak responses. This effect is particularly robust for the RSU-spiny cell populations (note the steep slopes in Fig. 6) but appears to be less so for the FSU-smooth cell populations, whose curves are flatter (see DISCUSSION).

The number of recorded units examined with GABA is smaller, particularly in the case of FSUs where adjacent whisker data are available for only two units, but results are consistent with those obtained with BMI. As shown in Fig. 7, GABA causes proportionately greater decreases in responses evoked by normally less effective stimuli, and this true for both classes of real and simulated neurons.

\section{Effects of BMI on between-whisker inhibitory interactions}

The preceding analyses were based on data obtained by moving the PW or adjacent whiskers one at a time and thus assess BMI's effects on excitatory receptive field properties. To evaluate its effects on inhibitory receptive field organization, we used a condition-test paradigm. Figure 8 shows receptive field properties of the same neuron as in Fig. 2, obtained by deflecting the PW alone (test stimulus) and in combination with prior deflection of each of its surrounding whiskers (condition-test stimuli). In the predrug condition, the neuron's excitatory receptive field was largely confined to the PW. Prior deflection of the gamma or D1 whisker produced noticeable inhibition of PW-evoked responses (an average reduction of $36 \%$ ), whereas deflection of $\mathrm{C} 2$ or $B 1$ produced less inhibition (an average $14 \%$ reduction). Application of $20 \mathrm{nA}$ of BMI led to 99 and $155 \%$ increases in the PW-alone ON and OFF responses, respectively, and an average $919 \%$ increase in adjacent whisker on responses. The PW responses that follow them, i.e., the conditioned-test responses, also are greatly enlarged, and surround inhibition averaged over the four adjacent whiskers decreased from 25 to $2 \%$.

Quantitative data for 18 RSUs and 5 FSUs are presented in Fig. 9. The conditioning stimulus consisted of deflections of the PW's caudally adjacent whisker in all eight directions and the test stimulus was deflection of the PW at its best angle. In the predrug condition, the PW responses of RSUs were reduced by approximately one-half as a result of the conditioning stimulus. Application of BMI caused a substantial loss of the adjacent whisker's inhibitory effects, producing condition-test ratios similar to the average value previously observed in thalamic neurons (rightmost bar in Fig. 9) (data from Simons and Carvell 1989). FSUs normally display less response suppression, and BMI reduced this also to roughly the same level as in the thalamus.

Simulated spiny and smooth cells were examined in the same way as their real counterparts. For these analyses, we examined model neurons whose overall response characteristics approximated those of the electrophysiologically studied RSUs and FSUs. The model neurons were comparably responsive to $\mathrm{PW}$ stimulation, had predrug condition-test ratios from 0.30 to 0.80 , and showed comparable increases in PW maximal angle oN responses when their incoming inhibitory synaptic weights were reduced (see legend to Fig. 9 ). With these constraints, the dependent variable, condition-test ratios under simulated BMI application, increased

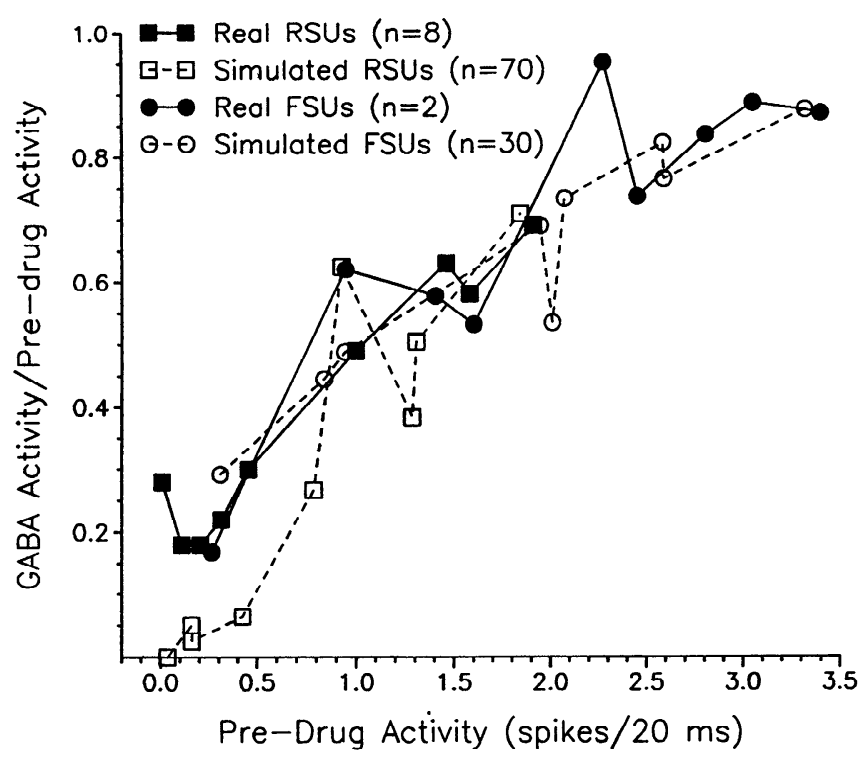

FIG. 7. GABA-induced changes in activity levels of real and simulated RSUs and FSUs. Conventions as in Fig. 6. GABA's effects were simulated by adding a constant hyperpolarizing voltage of $-0.73 \mathrm{mV}$ for spiny cells and $-0.31 \mathrm{mV}$ for smooth cells. These values were chosen because they reproduced the biological mean decrease in the RSU and FSU PW maximal angle ON responses, respectively; all other points represent independent variables. 


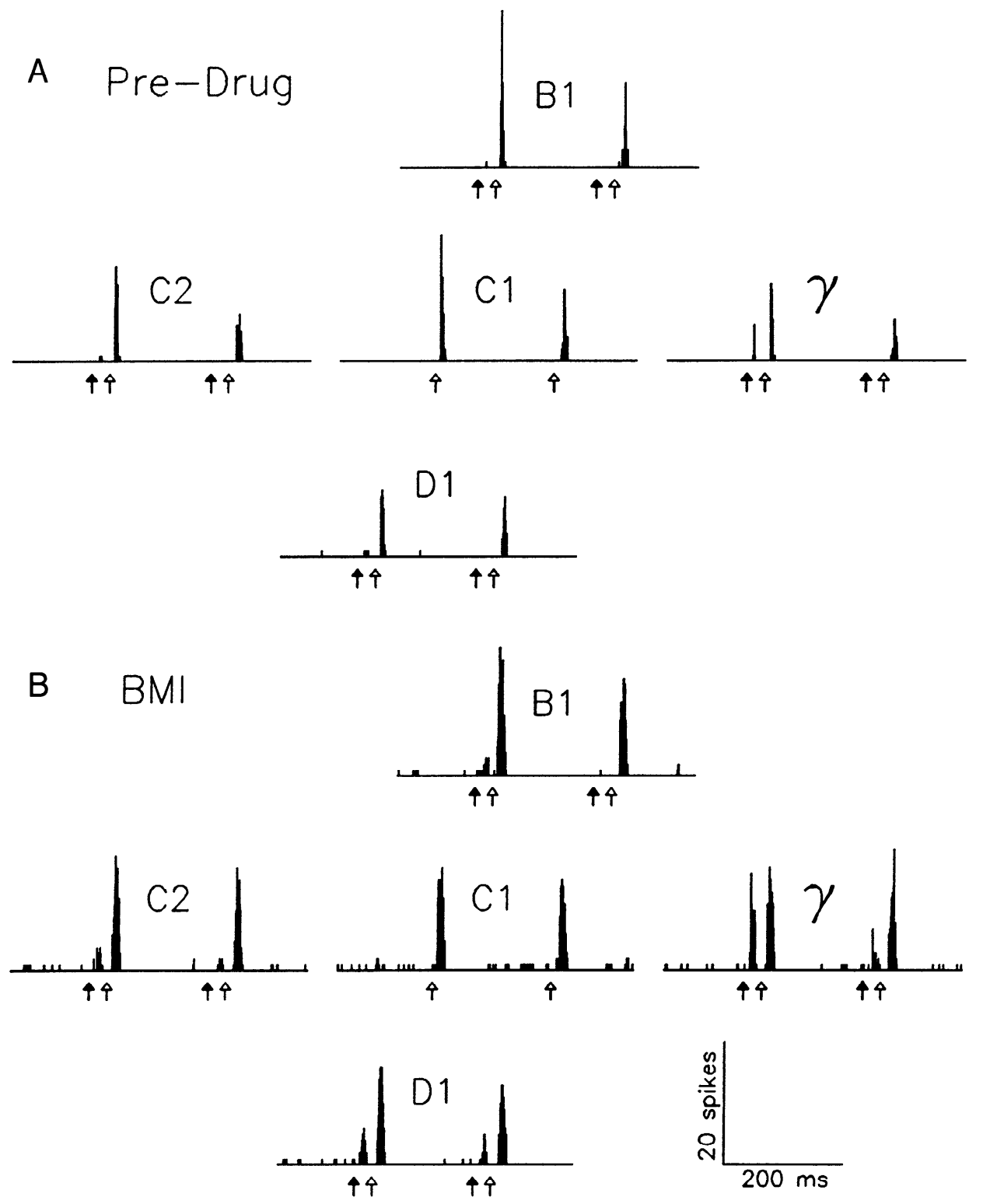

FIG. 8. Effect of BMI on excitatory and inhibitory receptive field characteristics of a barrel RSU. The unit is the same as that shown in Fig. 2, but it was studied here using the condition-test paradigm. Filled arrows: onsets and offsets of movement of the indicated adjacent whisker. Open arrows: onsets and offsets of deflection of $\mathrm{Cl}$, the PW. The BMI current was $20 \mathrm{nA}$.

substantially for both spiny and smooth cells. The disinhibition was even more pronounced than in the electrophysiologically studied units, and condition-test ratios were actually greater than those observed in thalamic neurons. This means that the size of the conditioned-test response, relative to the test alone, was larger than that observed in the cells that provided input to the model (see DISCUSSION).

The suppression of adjacent whisker responses by prior PW deflection is consistently greater than the suppressive effects of adjacent whisker deflections on PW responses. Such inhibition was reduced by BMI, too. Eleven RSUs had testable predrug adjacent whisker alone responses $(\geq 0.20$ spikes per stimulus). Their condition-test ratios averaged 0.19 , and these increased to 0.38 on BMI application. Surprisingly, FSUs displayed somewhat more PW-evoked inhibition in the presence of BMI; condition-test ratio actually decreased in three of the four units tested (an average of 0.50 was lowered to 0.24 ) and was unchanged in the other. We could not examine these effects in simulated neurons because of the absence of thalamic data that could serve as input, i.e., there were no thalamic spike trains obtained when the PW served as the conditioning stimulus.

\section{Mechanism of simulated drug effects}

For the model neurons, membrane potential is used to compute the probability that an action potential will occur during each millisecond. Examination of the model's behavior during simulated BMI application showed that decreasing inhibitory synaptic strengths led to a net increase in average membrane potential. Figure 10 shows the mean \pm SD of the membrane potentials at which simulated RSUs discharged spikes in response to PW or adjacent whisker movements. Data are presented with reference to the slope of the model RSUs' spike activation function. Also shown are the average response magnitudes in spikes per stimulus. In the case of normally weakly effective stimuli, e.g., adjacent whisker deflections, BMI-induced increases in membrane potential 


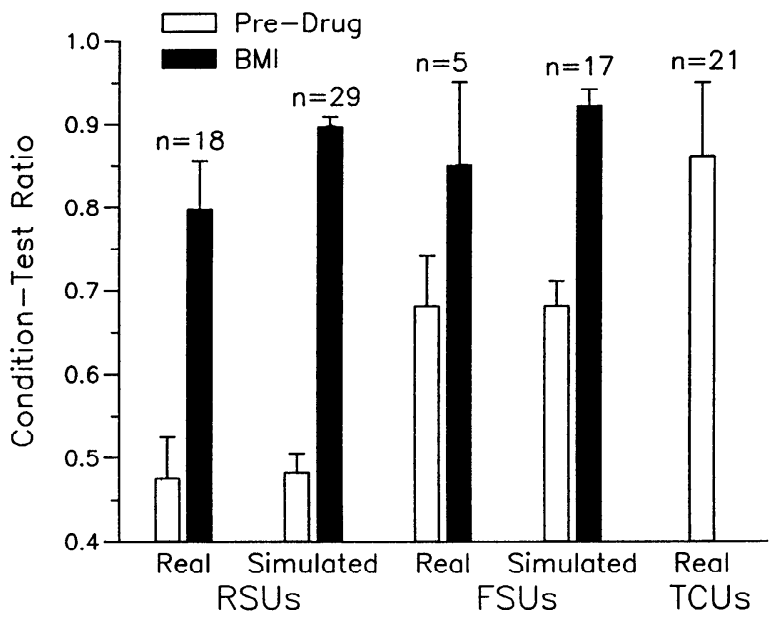

FIG. 9. Effect of BMI on surround inhibition in real and simulated barrel neurons. A condition-test ratio of 1.0 indicates no diminution of $\mathrm{PW}$ ON response by prior deflection of an adjacent whisker; 0.0 corresponds to complete response suppression. Simulated neurons were selected from the full populations of 70 spiny and 30 smooth cells on the basis of showing at least a 50 or $20 \%$ increase, respectively, in PW maximal on response with BMI, and by having initial condition-test ratios in the range of $0.3-$ 0.7 or $0.4-0.8$. For comparison, the mean condition-test ratio of 21 thalamocortical units (TCUs) is shown at right.

have the effect of moving the neuron away from the shallow slope and toward the steeper slope of the curve, where relatively small increases in membrane voltage result in relatively large increases in firing probability. Note that this resulted in a 3.7-fold increase in the number of evoked spikes. For stronger inputs, such as those associated with PW deflection onsets, neurons were already positioned nearer the top of the curve so that BMI-induced increases in membrane potential resulted in comparatively smaller increases in spike output (a 2.3-fold increase). It is important to note that stimulus-evoked responses of the model neurons were always considerably less than the theoretical maximum of 5.0 spikes per stimulus (see legend). Simulation of GABA application, by adding a constant hyperpolarizing voltage, moved neurons in the opposite direction along the function's slope (not shown).

\section{DISCUSSION}

\section{Disinhibition in real and simulated barrels}

One purpose of the present study was to test the ability of a previously constructed computer model (Kyriazi and Simons 1993) to predict quantitatively the effects of GABA and BMI on real barrel neurons. Established initially to reproduce barrel RSU and FSU responses to PW stimulation, the model has accurately predicted excitatory responses to adjacent whisker deflections, inhibition produced by condition-test stimuli, decreases in ON-to-OFF ratios with increases in stimulus duration, and now, alterations in a variety of receptive field properties induced by BMI and GABA application. We emphasize that, in simulating BMI or GABA application, the model neurons' inhibitory weights or membrane potentials were adjusted by an amount sufficient to reproduce only the average change in on response magnitude at the best deflection angle; all other simulated responses were independent variables. Although not simulated in biophysical detail, the model neurons accurately predicted the response magnitudes of these independent variables, capturing the drug-induced disproportionate changes to strongly versus weakly effective stimuli. Similar biological results can be seen in a study of BMI's effects on forearm receptive field sizes in cat somatosensory cortex (Alloway et al.

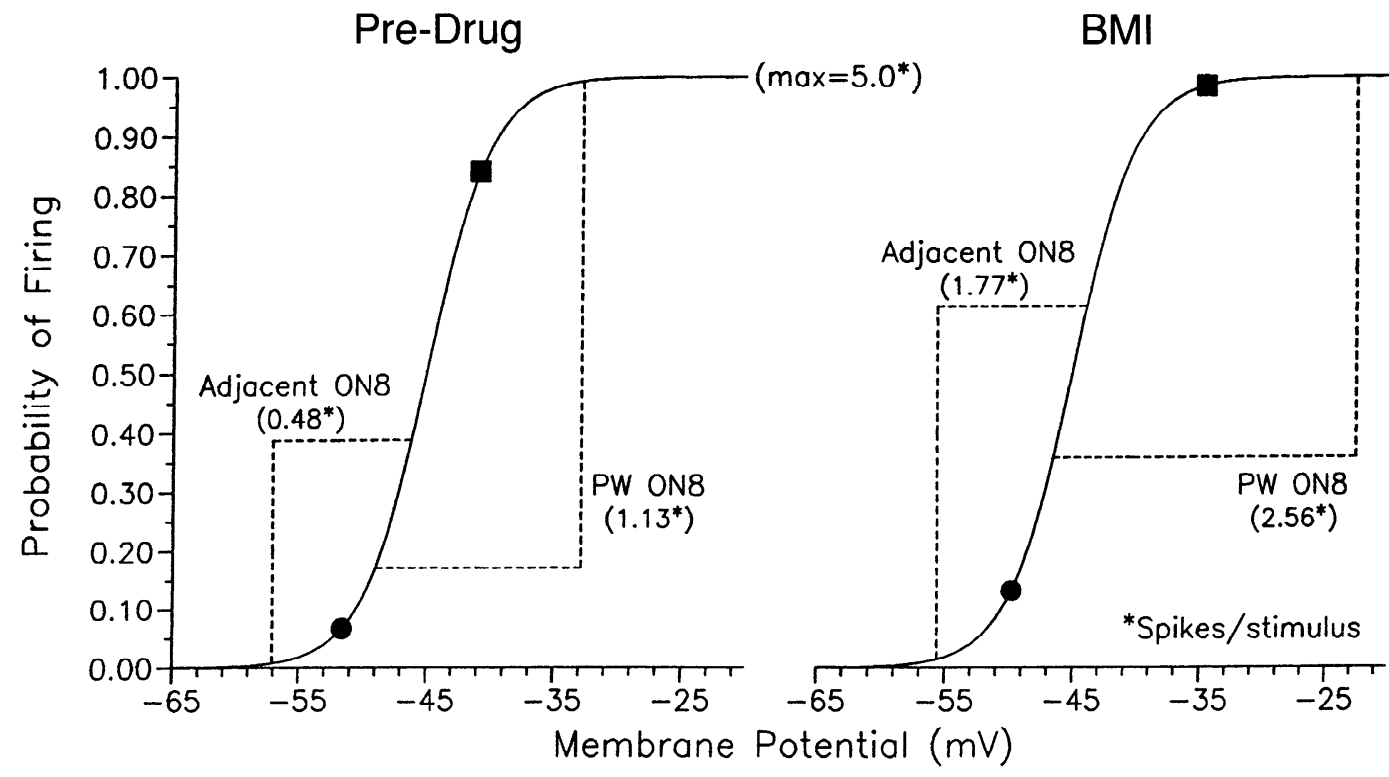

FIG. 10. BMI's effect on simulated spiny cell membrane potentials. The sigmoid curves, identical in both panels, plot the spike activation function relating membrane potential to probability of spiny cell spike discharge. Denoted points on each curve indicate the mean membrane potential at which spike discharge actually occurred ( $\mathbf{m}$ : PW ON responses averaged over 8 angles; $\bullet$ : adjacent whisker on responses averaged over 8 angles). Dashed lines: 1 SD. Left: data for the predrug condition. Right: data for the same responses under simulated BMI. Numbers: associated response magnitudes in spikes per stimulus. The value of 5.0 at the curve's upper limit (probability of 1.0) is the largest response that can occur during the 20 -ms response window, because spiny cells are assigned an absolute refractory period of $4 \mathrm{~ms}$. 
1989). In fact, expression of the quantitative data presented in Fig. 5 of that study in terms of proportional changes plotted against predrug values yields data points that fall along the curve for our real and simulated RSU data points in Fig. 6.

It has been suggested that BMI-induced alterations in receptive field propertics of visual cortical neurons are due to spread of the drug onto many neighboring neurons in addition to the cell being studied electrophysiologically (Ferster 1987; Nelson et al. 1994). In the present study, the microiontophoretic barrels were within $10 \mu \mathrm{m}$ of the tip of the recording electrode, and, perhaps as a result, small BMI ejection currents were typically effective. Although it is likely that the drug still diffused onto nearby neurons, the fact that quantitatively similar changes in response properties were produced in the model neurons, where only a single postsynaptic cell was manipulated, suggests that direct effects of the drug on neighboring cells are not required to account for the electrophysiological results.

On the basis of these findings for real and simulated barrel neurons, we propose that in the electrophysiological experiments BMI produced a general increase in excitability that acted in conjunction with neuronal nonlinearities to produce the observed disproportionate effects. By blocking synaptically induced $\mathrm{Cl}^{-}$conductance increases, BMI would reduce the tendency of a cell to remain near its resting potential, and the cell, once excited, would be more likely to remain depolarized for a longer time, and thus more likely to fire. Recently it has been shown that normal orientation tuning of visual cortical neurons can be maintained in the absence of both $\mathrm{GABA}_{\mathrm{A}^{-}}$and $\mathrm{GABA}_{\mathrm{B}}$-mediated inhibition when somal membrane potentials are kept at near normal levels by steady application of hyperpolarizing current (Nelson et al. 1994). Accordingly, it should be possible to effect changes in receptive field properties similar to those observed here with BMI application simply by depolarizing the cell membrane, perhaps using intracellular injections of depolarizing current or extracellular iontophoresis of $\mathrm{K}^{+}$.

\section{Inhibition affects spatiotemporal summation of thalamocortical inputs}

The present findings demonstrate that blockade of $\mathrm{GABA}_{\mathrm{A}}$ receptors by $B M I$ leads to enlarged receptive fields, broader angular tuning, smaller differences between ON and OFF responses, and diminished surround and "in-field" inhibition. GABA has the reverse effects. Comparable effects have been observed in cat vibrissa cortex, which lacks barrels; bicuculline or picrotoxin decreases directional tuning and diminishes neighboring whisker-evoked condition-test inhibition (Batuev et al. 1989). A particularly interesting finding in the study by Batuev et al. is that application of glutamate at a site $200-300 \mu \mathrm{m}$ distant from the recording electrode counteracted the effects of locally applied picrotoxin and, by itself, produced an enhancement of directional tuning similar to what we observed with the use of locally applied GABA. These findings indicate that at least some inhibitory receptive field properties of vibrissa cortex neurons are mediated locally.

In many respects, BMI renders the responses of barrel neurons, particularly RSUs, similar to those of the neurons in the ventrobasal thalamic nucleus, the major source of afferent input to the barrel. Barrel neurons can receive convergent thalamic excitation either directly via monosynaptic thalamocortical synapses or secondarily by means of excitatory connections mediated by spiny neurons within the barrel. We propose that the effects of BMI can be explained by the aforementioned depolarization-induced alterations in the spatiotemporal summation of such convergent thalamic drive. For example, RSUs are normally somewhat less selective for deflection angle than thalamic neurons (Simons and Carvell 1989), which are themselves considerably less selective than the primary afferent fibers innervating the whisker follicles (Lichtenstein et al. 1990; Zucker and Welker 1969). The even greater broadening of the polar plots with BMI application would thus reflect spatial convergence onto individual barrel neurons from multiple thalamic neurons, each having a slightly different directional profile. Similarly, compared with thalamic neurons, RSUs normally display greater response differentials to stimulus onsets versus offsets. In the vibrissa/barrel system, off responses are less vigorous and more temporally dispersed than on responses, and thus more dependent on temporal summation within barrel circuitry (Kyriazi et al. 1994). In the present study BMI enhanced OFF responses more than ON responses, presumably by producing a net increase in the duration of stimulusevoked depolarizations that in turn allowed for greater temporal summation.

Compared with thalamic neurons and cortical RSUs, fastspike barrel neurons have the poorest angular tuning and the smallest ON-to-OFF ratios. These properties probably reflect the generally high level of responsiveness that FSUs display normally and the related finding that smooth barrel neurons, which discharge fast spikes, receive thalamocortical synapses on proximal dendritic shafts and cell soma (Keller and White 1987). By contrast, spiny cells receive the vast majority of their thalamic inputs on spines, distributed along the length of their dendrites (White and Rock 1980), and this may contribute to the greater nonlinearity of the RSU response. Thus the stimulus-evoked discharges of FSUs may be less dependent on spatiotemporal summation of thalamic inputs than those of RSUs (Kyriazi et al. 1994). This would account for the present finding that FSU responses were quantitatively less strongly affected by BMI.

The data of Fig. 6 suggest that BMI might affect RSUs and FSUs differently with regard to weak versus strong responses. Fast-spike neurons repolarize especially rapidly and display only brief hyperpolarizations following action potential discharge (Kawaguchi 1993; McCormick et al. 1985), and perhaps the apparent difference in the FSU and RSU curves of Fig. 6 reflects such intrinsic differences between these two cell types. As noted in the RESULTS section, however, the largest differences in the RSU and FSU curves occur at points corresponding to spontaneous, not stimulusevoked, activity. Thus it may be equally plausible that the RSU-spiny cell and FSU-smooth cell data represent a continuum related to pre-BMI responsiveness.

\section{Origins of receptive field enlargements}

BMI-induced increases in receptive field size, measured here as larger adjacent whisker-evoked responses, may re- 
flect an enhanced effectiveness of spatiotemporal summation of EPSPs arising from convergent inputs from thalamic neurons having multiwhisker receptive fields. In our simulations, thalamic input arises solely from neurons in the homologous barreloid, and all network connections occur among neurons within the (single) cortical barrel. Thus thalamic neurons with multiwhisker receptive fields make up the only source of adjacent whisker input to the simulated neurons, a simplification of the connectivitives that are likely to occur in real barrels ( see Keller 1995). Nevertheless, when inhibitory synaptic strengths were reduced, model neurons displayed increases in adjacent whisker-evoked responses that quantitatively matched those of real barrel neurons subjected to BMI. Thus the unmasking of horizontal connections linking nearby barrels (columns) is not a necessary condition to account for findings in real barrels. As illustrated in Figs. 1 and 8, the time course of RSU adjacent whisker responses was similar to that evoked by PW stimulation, and, significantly, BMI-induced increases were observed in the earliest components of the adjacent whisker response, that is, at a latency consistent with direct thalamocortical input. Small increases in longer-latency responses, such as in the B2 and C3 PSTHs of Fig. $1 C$, were also occasionally observed, and these may reflect column-to-column interactions such as those described in urethan-anesthetized animals (Armstrong-James et al. 1991), where long-latency, long-duration adjacent whisker responses are particularly prominent ( $\mathrm{Si}-$ mons et al. 1992).

We wish to emphasize that the present findings and our interpretation of them apply only to layer IV barrel neurons, which are likely to be strongly activated by thalamic afferents. Studies of BMI's effects on somatosensory cortex have consistently reported increases in receptive field size, using test stimuli over a much greater skin area than those used here (Alloway et al. 1989; Dykes et al. 1984). Single thalamocortical axons are known to ramify extensively in the tangential plane (Garraghty and Sur 1990; Garraghty et al. 1989 Landry and Deschenes 1981), so that the receptive field expansions seen at middle cortical depths in other species might also reflect BMI's unmasking of less numerous and/or less synchronously active thalamic signals. For neurons in other layers, intercolumnar connections may be the substrate for normally subthreshold inputs that become expressed with BMI application.

\section{Contribution of inhibition to cortical receptive field properties}

An important finding in this study and in previous studies of somatosensory cortex is that FSUs, corresponding to at least one class of GABAergic inhibitory interneurons (Kawaguchi 1993; Kawaguchi and Kubota 1993; McCormick et al. 1985), characteristically display high rates of background and stimulus-evoked activity (Mountcastle et al. 1969; Simons 1978; Swadlow 1989). Like GABA microiontophoresis, tonic inhibition produced by FSUs would more strongly suppress responses to stimuli evoking only weak excitatory afferent drive. It thereby normally acts to constrain receptive field size and enhance angular tuning. Such effects in RSUs have been found to accompany increases in FSU 'background" activity produced by small-amplitude, random noise vibration of neighboring whiskers (Brumberg and Simons 1994).

Whisker stimuli cvoke an initial, brief excitation followed a few milliseconds later by a longer-lasting inhibition (Carvell and Simons 1988; Hellweg et al. 1977; and see Agmon and Connors 1992). BMI's antagonism of the latter presumably caused the marked reduction of betweenwhisker inhibition observed in the present study. This was the case for both real and simulated neurons. The latter, however, displayed a greater loss of surround inhibition than the electrophysiologically studied units. Neurons in real barrels are subject to both $\mathrm{GABA}_{\mathrm{A}^{-}}$and $\mathrm{GABA}_{\mathrm{B}}$-mediated inhibition (unpublished observations), and thus only a portion of their inhibitory input was affected by the application of $B M I$. The model neurons, on the other hand, have only one type of inhibition (a fast-acting $\mathrm{GABA}_{\mathrm{A}}$ type), all of which was affected by simulated antagonist application. Consequently, combined use of BMI and phaclofen, a GABA antagonist, should produce, in real barrels, an even greater loss of surround inhibition than observed in the present study. Under such conditions, barrel neurons might even display less surround inhibition than their thalamic inputs, as observed in the simulations. When both fast- and slowacting inhibition are diminished, the postexcitatory inhibition following the conditioning stimulus would be reduced, and the excitatory effects of the (now larger) adjacent whisker response might actually potentiate the response to the test stimulus.

We thank Q. Zhou for participating in some of the early experiments.

This work was supported by National Institute of Neurological Disorders and Stroke Grant NS-19950 and by Pittsburgh Supcrcomputing Center Grant 1-P4I-RR06009 from the NIH National Center for Research Resources.

Address reprint requests to $\mathrm{H}$. Kyriazi.

Received 4 April 1995; accepted in final form 14 September 1995.

\section{REFERENCES}

Agmon, A. And Connors, B. W. Correlation between intrinsic firing patterns and thalamocortical synaptic responses of neurons in mouse barrel cortex. J. Neurosci. 12: 319-329, 1992.

AllowAY, K. D. AND BURTON, H. Bicuculline-induced alterations in neuronal responses to controlled tactile stimuli in the second somatosensory cortex of the cat: a microiontophoretic study. Somatosens. Res. 3: 197$211,1986$.

Alloway, K. D., Rosenthal, P., and Burton, H. Quantitative mcasurements of receptive field changes during antagonism of GABAergic transmission in primary somatosensory cortex of cats. Exp. Brain Res. 78: 514-532, 1989.

Andersen, P., Dingledine, R., Gjerstad, L., Langmoen, I. A., and MosFELDT LAUREN, A. Two different responses of hippocampal pyramidal cells to application of gamma-amino butyric acid. J. Physiol. Lond. 305: 279-296, 1980

Armstrong-James, M., Callahan, C. A., And Friedman, M. A. Thalamocortical processing of vibrissal information in the rat. I. Intracortical origins of surround but not centre-receptive fields of layer IV neurones in the rat S1 barrel field cortex. J. Comp. Neurol. 303: 193-210, 1991.

ARMSTRONG-JAMES, M. AND Fox, K. Spatiotemporal convergence and divergence in the rat SI "barrel"' cortex. J. Comp. Neurol. 263: 265-281, 1987.

Armstrong-James, M., Fox, K., And Millar, J. M. A method for etching the tips of carbon fibre microelectrodes. J. Neurosci. Methods 2: $431-$ 432, 1980.

Batuev, A. S., Alexandrov, A. A., Scheynikov, N. A., Kcharazia, V. N., AND AN, C. C. The role of inhibitory processes in the formation 
of functional properties of neurons in vibrissal projection zone of the cat somatosensory cortex. Exp. Brain Res. 76: 198-206, 1989.

BRUMBERG, J. C. AND SimONS, D. J. Receptive field organization and spatial summation of whisker inputs in rat barrel cortex. Soc. Neurosci. Abstr. 19: 107, 1994.

Carvell, G. E. And Simons, D. J. Membrane potential changes in rat SmI cortical neurons evoked by controlled stimulation of mystacial vibrissae. Brain Res. 448: 186-191, 1988.

Connors, B. W., Malenka, R. C., And Silva, L. R. Two inhibitory postsynaptic potentials, and $\mathrm{GABA}_{\mathrm{A}}$ and $\mathrm{GABA}_{\mathrm{B}}$ receptor-mediated responses in neocortex of rat and cat. J. Physiol. Lond. 406: 443-468, 1988.

Douglas, R. J., Martin, K. A. C., And Whitteridge, D. An intracellular analysis of the visual responses of neurones in cat visual cortex. $J$. Physiol. Lond. 440: 659-696, 1991.

Dykes, R. W., Landry, P., Metherate, R., and Hicks, T. P. Functional role of GABA in cat primary somatosensory cortex: shaping receptive fields of cortical neurons. J. Neurophysiol. 52: 1066-1093, 1984.

FERSTER, D. Orientation selectivity of synaptic potentials in neurons of cat primary visual cortex. J. Neurosci. 6: 1284-1301, 1986.

FERSTER, D. Origin of orientation-selective EPSPs in simple cells of cat visual cortex. J. Neurosci. 7: 1780-1791, 1987.

GARDNER, E. P. AND COSTANZO, R. M. Temporal integration of multiplepoint stimuli in primary somatosensory cortical receptive fields of alert monkeys. J. Neurophysiol. 43: 444-468, 1980.

Garraghty, P. E., Pons, T. P., Sur, M., And KaAs, J. H. The arbors of axons terminating in middle cortical layers of somatosensory area $3 \mathrm{~b}$ in owl monkeys. Somatosens. Mot. Res. 6: 401-411, 1989.

GaRRAGHTY, P. E. AND SUR, M. Morphology of single intracellularly stained axons terminating in area $3 \mathrm{~b}$ of macaque monkeys. J. Comp. Neurol. 294: 583-593, 1990.

Hellweg, F. C., Schultz, W., And Creutzfeldt, O. D. Extracellular and intracellular recordings from cat's cortical whisker projection area: thalamocortical response transformation. J. Neurophysiol. 40: 463-479, 1977.

JACOBS, K. M. AND Donoghue, J. P. Reshaping the cortical motor map by unmasking latent intracortical connections. Science Wash. DC 251: 944$947,1991$.

KAWAGUCHI, Y. Groupings of nonpyramidal and pyramidal cells with specific physiological and morphological characteristics in rat frontal cortex. J. Neurophysiol. 69: 416-431, 1993.

KAWAGUCHI, Y. AND KUBOTA, Y. Corrclation of physiological subgroupings of nonpyramidal cells with parvalbumin- and calbindinD28k-immunoreactive neurons in layer V of rat frontal cortex. J. Neurophysiol. 70: 387396, 1993.

Keller, A. Synaptic organization of the barrel cortex. In: Cerebral Cortex, cdited by E. G. Jones and I. T. Diamond. New York: Plenum, 1995, vol. 11, p. $221-262$.

Keller, A. AND White, E. L. Synaptic organization of GABAergic neurons in mouse SmI cortex. J. Comp. Neurol. 262: 1-12, 1987.

Kyriazi, H. T., Carvell, G. E., and Simons, D. J. off response transformations in the whisker/barrel system. J. Neurophysiol. 72: 392-401, 1994.

KYRIAZI, H. T. AND SIMONS, D. J. Thalamocortical response transformations in simulated whisker barrels. J. Neurosci. 13: 1601-1615, 1993.

LANDRY, P. AND DESCHENES, M. Intracortical arborizations and receptive fields of identified ventrobasal thalamocortical afferents to the primary somatic sensory cortex in the cat. J. Comp. Neurol. 199: 345-371, 1981.

LASKIN, S. E. AND SPENCER, W. A. Cutaneous masking. II. Geometry of excitatory and inhibitory receptive fields of single units in somatosensory cortex of the cat. J. Neurophysiol. 42: 1061-1082, 1979.

Lichtenstein, S. H., Carvell, G. E., And Simons, D. J. Responses of rat trigeminal ganglion neurons to movements of vibrissae in different directions. Somatosens. Mot. Res. 7: 47-65, 1990.

McCasland, J. S., Carvell, G. E., Simons, D. J., and Woolsey, T. A. Functional asymmetries in the rodent barrel cortex. Somatosens. Mot. Res. 8: $111-116,1991$.

McCasland, J. S., Hibbard, L. S., Kalmbach, S., and Woolsey, T. A. Cellular maps of metabolic activity in antigenically identified neurons: a 2-deoxyglucose/immunostaining approach to barrel field circuitry. Soc. Neurosci. Abstr. 18: 1546, 1992.

McCormick, D. A., Connors, B. W., Lighthall, J. W., And Prince, D. A. Comparative electrophysiology of pyramidal and sparsely spiny stellate neurons of the neocortex. J. Neurophysiol. 54: 782-806, 1985.

Mountcastle, V. B., Talbot, W. H., Sakata, H., and Hyvarinen, J. Cortical neuronal mechanisms in flutter-vibration studied in unanesthetized monkeys. Neuronal periodicity and frequency discrimination. $J$. Neurophysiol. 32: 452-484, 1969.

Nelson, S., Toth, L., Sheth, B., AND Sur, M. Orientation selectivity of cortical neurons during intracellular blockage of inhibition. Science Wash. DC 265: 774-777, 1994.

Sillito, A. M. The contribution of inhibitory mechanisms to the receptive field properties of neurons in the striate cortex of the cat. I. Physiol. Lond. 250: 305-329, 1975.

SIMONS, D. J. Response properties of vibrissa units in the rat SI somatosensory neocortex. J. Neurophysiol. 41: 798-820, 1978.

SimONS, D. J. Multi-whisker stimulation and its effects on vibrissa units in rat SmI barrel cortex. Brain Res. 276: 178-182, 1983.

Simons, D. J. Temporal and spatial integration in the rat SI vibrissa cortex. J. Neurophysiol. 54: 615-635, 1985.

Simons, D. J. AND CARVELl, G. E. Thalamocortical response transformation in the rat vibrissa/barrel system. J. Neurophysiol. 61: 311-330, 1989.

Simons, D. J., Carvell, G. E., Hershey, A. E., and Bryant, D. P. Responses of barrel cortex neurons in awake rats and effects of urethane ancsthesia. Exp. Brain Res. 91: 259-272, 1992.

SimONS, D. J. AND LAND, P. W. A reliable technique for marking the location of extracellular recording sites using glass micropipettes. Neurosci. Lett. 81: $100-104,1987$.

SIMONS, D. J. AND WoOLSEY, T. A. Functional organization in mouse barrel cortex. Brain Res. 165: 327-332, 1979.

SwADLOW, H. A. Efferent neurons and suspected interneurons in S-1 vibrissa cortex of the awake rabbit: receptive fields and axonal properties. J. Neurophysiol. 62: 288-308, 1989.

WELKER, C. Receptive fields of barrels in the somatosensory neocortex of the rat. J. Comp. Neurol. 166: 173-189, 1976.

WHITE, E. L. Thalamocortical synaptic relations: a review with emphasis on projections of specific thalamic nuclei to the primary sensory areas of the neocortex. Brain Res. Rev. 1: 275-311, 1979.

White, E. L. Cortical Circuits: Synaptic Organization of the Cerebral Cortex. Boston, MA: Birkhäuser, 1989.

WhITE, E. L. AND Rock, M. P. Three-dimensional aspects and synaptic relationships of a Golgi-impregnated spiny stellate cell reconstructed from serial thin sections. J. Neurocytol. 9: 615-636, 1980.

WOOLSEY, T. A. AND VAN DER LOOS, H. The structural organization of layer IV in the somatosensory region (SI) of mouse cerebral cortex. Brain Res. 17: 205-242, 1970.

ZUCKER, E. AND WELKER, W. I. Coding of somatic sensory input by vibrissal neurons in the rat's trigeminal ganglion. Brain Res. 12: 138-156, 1969. 